\title{
Local Injection of Growth Hormone for Temporomandibular Joint Osteoarthritis
}

\author{
Soo-Min $\mathrm{Ok}^{1,2}$, Jin-Hwa Kim², Ji-Su Kim², Eun-gyo Jeong², Yang Mi Park², Hye-Mi Jeon², \\ Jun-Young $\mathrm{Heo}^{1}$, Yong-Woo $\mathrm{Ahn}^{1,2}$, Sun-Nyoung Yu${ }^{3}$, Hae Ryoun Park ${ }^{4}$, \\ Kyung-Hee $\mathrm{Kim}^{5}$, Soon-Cheol $\mathrm{Ahn}^{3}$, and Sung-Hee Jeong ${ }^{1,2}$ \\ ${ }^{1}$ Department of Oral Medicine, Dental Research Institute, Pusan National University Dental Hospital, Yangsan; \\ ${ }^{2}$ Department of Oral Medicine, Dental and Life Science Institute, Pusan National University School of Dentistry, Yangsan; \\ ${ }^{3}$ Department of Microbiology \& Immunology, Pusan National University School of Medicine, Yangsan; \\ ${ }^{4}$ Department of Oral Pathology, Pusan National University School of Dentistry, Yangsan; \\ ${ }^{5}$ Deptartment of Oral Medicine, Busan Paik Hospital, Inje University, Busan, Korea.
}

Purpose: Osteoarthritis (OA) of the temporomandibular joint (TMJ) elicits cartilage and subchondral bone defects. Growth hormone $(\mathrm{GH})$ promotes chondrocyte growth. The aim of this study was to evaluate the efficacy of intra-articular injections of GH to treat TMJ-OA.

Materials and Methods: Monosodium iodoacetate (MIA) was used to induce OA in the TMJs of rats. After confirming the induction of OA, recombinant human GH was injected into the articular cavities of rats. Concentrations of GH and IGF-1 were measured in the blood and synovial fluid, and OA grades of cartilage and subchondral bone degradation were recorded by histological examination and micro-computed tomography.

Results: MIA-induced OA in the rat TMJ upregulated insulin-like growth factor-1 (IGF-1) rather than GH levels. GH and IGF-1 concentrations were increased after local injection of GH, compared with controls. Locally injected GH lowered osteoarthritic scores in the cartilage and subchondral bone of the TMJ.

Conclusion: Intra-articular injection of GH improved OA scores in rat TMJs in both cartilage and subchondral bone of the condyles without affecting condylar bone growth. These results suggest that intra-articular injection of human GH could be a suitable treatment option for TMJ-OA patients in the future.

Key Words: Growth hormone, intraarticular injection, osteoarthritis, temporomandibular joint

\section{INTRODUCTION}

Osteoarthritis (OA) is a chronic inflammatory disease that progressively debilitates joints and the surrounding tissue. OA lim-

Received: September 26, 2019 Revised: February 9, 2020

Accepted: February 18, 2020

Corresponding author: Sung-Hee Jeong, DMD, MSD, PhD, Department of Oral Medicine, Dental Research Institute, Dental and Life Science Institute, Pusan National University School of Dentistry, 49 Busandaehak-ro, Mulgeum-eup, Yangsan 50612, Korea.

Tel: 82-55-360-5242, Fax: 82-55-360-5238, E-mail: drcookie@pusan.ac.kr

-The authors have no potential conflicts of interest to disclose.

(c) Copyright: Yonsei University College of Medicine 2020

This is an Open Access article distributed under the terms of the Creative Commons Attribution Non-Commercial License (https://creativecommons.org/licenses/ by-nc/4.0) which permits unrestricted non-commercial use, distribution, and reproduction in any medium, provided the original work is properly cited. its movement, distorts joint shape, and causes joint pain. OA of the temporomandibular joint (TMJ-OA) poses difficulties with chewing, limits mouth opening, and causes facial asymmetry, ${ }^{1}$ and nutritional imbalances due to pain and masticatory dysfunction may impede growth and development. ${ }^{2,3}$

One study investigating the demographics of patients visiting an orofacial pain clinic in South Korea noted an increase in young TMD patients with OA from $9 \%$ in 2000 to $39 \%$ in $2008 .{ }^{4} \mathrm{TMJ}-\mathrm{OA}$ that starts at a young age inevitably leads to facial deformity and malocclusion. ${ }^{5}$ To solve these problems, orthodontic treatment with orthognathic surgery is generally required. However, sudden changes in occlusion can aggravate TMJ-OA and worsen the problem, thus treatment cannot be started until TMJ-OA is completely cured. ${ }^{6}$ Therefore, early treatment before irreversible bony changes occur is needed.

Human growth hormone (hGH) stimulates growth by trig- 
gering active chondrocyte growth at the postnatal epiphyseal growth plates of the long bone. ${ }^{7}$ Exerting growth promoting effect on chondrocytes, hGH has been suggested as a treatment strategy for OA. ${ }^{8} \mathrm{hGH}$ showed a positive effect in the very early healing phase of an osteochondral defect in an animal model. ${ }^{9}$ Reinecke, et al. ${ }^{10}$ concluded that hGH could be used as a potential treatment option for OA because of its anabolic effect on joints. Additionally, hGH could be used with the purpose of increasing cartilage metabolism and chondrocyte proliferation. ${ }^{11}$ Therefore, use of hGH to treat OA could be optimized by avoiding systemic administration and using it locally in only problematic TMJ-OA sites.

Growth hormone (GH) has been shown to be involved in the proliferation of cartilage by stimulating insulin-like growth factor 1 (IGF-1) production in chondrocytes and modulating the growth metabolism of cartilage. ${ }^{12,13}$ Accordingly, IGF-1 could modify the progression of OA via cartilage growth. ${ }^{14} \mathrm{GH}$ regulates cell growth using IGF-1 as a mediator. Immunolocalized studies have confirmed that when GH is directly injected into the growth plate, IGF-1 levels in the growth plate are increased. ${ }^{15}$ This mechanism was reflected as increases in the mRNA and protein levels of IGF-1 when GH was systemically administered.$^{16}$ Therefore, IGF-1 and hGH were confirmed to be capable of being utilized as monitoring markers of $\mathrm{OA} .{ }^{17}$

This study sought to document changes that occurred after the local application of GH in TMJ-OA chemically induced in rats by monosodium iodoacetate (MIA) and to evaluate intraarticular injections of hGH as a suitable treatment option for TMJ-OA patients in the future.

\section{MATERIALS AND METHODS}

\section{Animals}

Seventeen 4-week-old male Sprague-Dawley rats (Koatech, Pyeongtaeck, Korea), weighing 90-100 g, were used, providing a total of 34 TMJs. Animals were housed one rat per cage at 21$22^{\circ} \mathrm{C}$ with a 12 -h light/12-h dark cycle. All experiments were approved by the Animal Ethics Committee of Pusan National University (PNU-2014-0518) and performed according to Guidelines for the Care and Use of Laboratory Animals.

\section{Study design}

TMJ-OA was induced in 4-week-old experimental animals as described in a previous paper..$^{18}$ Briefly, $50 \mathrm{~mL}$ of solution [monosodium iodoacetate (MIA) $1 \mathrm{mg}$ ] was injected into the TMJ bilaterally, and 2 weeks later, typical OA stages were observed. After the animals were anesthetized with inhaled isoflurane, a 50- $\mu \mathrm{L}$ intra-articular injection of MIA $1 \mathrm{mg}$ (Sigma, St. Louis, MO, USA) was administered in both condyles. After 2 weeks, $50 \mu \mathrm{L}$ of phosphate buffered saline (PBS) was injected into the articular space of the right condyle and $50 \mu \mathrm{L}$ of a solution of $500 \mu \mathrm{g}$ of recombinant human growth hormone
(rhGH) (Pfizer, New York, NY, USA) in PBS was injected into the articular space of the left condyle. The procedures were repeated twice a week at 3-day intervals for a total of four injections. The animals were euthanized after 2 or $4 \mathrm{rhGH}$ injections (Fig. 1). OA was induced in all experimental animals using MIA except in two healthy controls. Ten randomly selected rats were injected with $\mathrm{GH}(\mathrm{OA}+\mathrm{GH}$ injected) and PBS $(\mathrm{OA}+\mathrm{PBS}$ injected) in each TMJ according to the experimental protocol, and the remaining five were left to self-heal after $\mathrm{OA}$ induction (OA-induced only). The bilateral condyles were collected for histological observation, and serum and synovial fluid of the TMJ were collected. Synovial fluid was collected from both condyles by a lavage of $50 \mu \mathrm{L}$ of sterile saline infused into the joint and withdrawn by syringe.

\section{Micro-computed tomography}

Under intraperitoneal anesthesia with Zoletil $0.6 \mathrm{~mL} / \mathrm{kg}$ (Virbac Korea, Seoul, Korea) and Rompun $0.4 \mathrm{~mL} / \mathrm{kg}$ (Bayer Korea, Seoul, Korea), the rats were placed on a volumetric computed tomography (CT) scanner (NFR-MXSCAN-G90; NanoFocusRay, Iksan, Korea), a micro-CT system designed to minimize motion artifacts and to maintain respiratory anesthesia during scanning to obtain CT images of a live rat. The system was set to $70 \mathrm{kV}$ and $60 \mathrm{~mA}$. A micro-CT scan was performed three times to measure condylar length and to check for MIA-induced arthritis. The CT images were obtained at the following time points: just before starting GH injection (CT1, 6-weekold) and after two (CT2, 7-week-old) or four (CT3, 8-week-old) GH treatments (Fig. 1).

For the measurement of condylar length, a straight line was drawn between two points anatomically situated in the lowest position on two-dimensional reconstructed images. Mandibular head length was measured by drawing a perpendicular line from the highest point of the condylar process toward the former line. ${ }^{19}$

\section{Subchondral bone OA score}

For the scoring of radiographic signs of OA, two individuals participated in this experiment independently. The lateral, central, and medial parts of the condyle were surveyed and graded as previously described.$^{20}$ In this study, only erosive changes of the condyle were recorded before and after GH treatment. Subchondral bone OA scores were classified as follows: None, 0; Minor, 1; Moderate, 2; or Severe, 3 (Fig. 2A-D).

\section{Histological observation}

For histological observation, the bilateral condyles of each rat were dissected, immersed in $10 \%$ formaldehyde, and decalcified in Plank-Rychlo solution. Each trimmed specimen was processed following a standard procedure for paraffin preparation: hydration in a graded series of ethanol, clearance by xylene, and embedding in paraffin. The 4-mm-thick frontal sections were deparaffined and visualized by Masson's tri- 

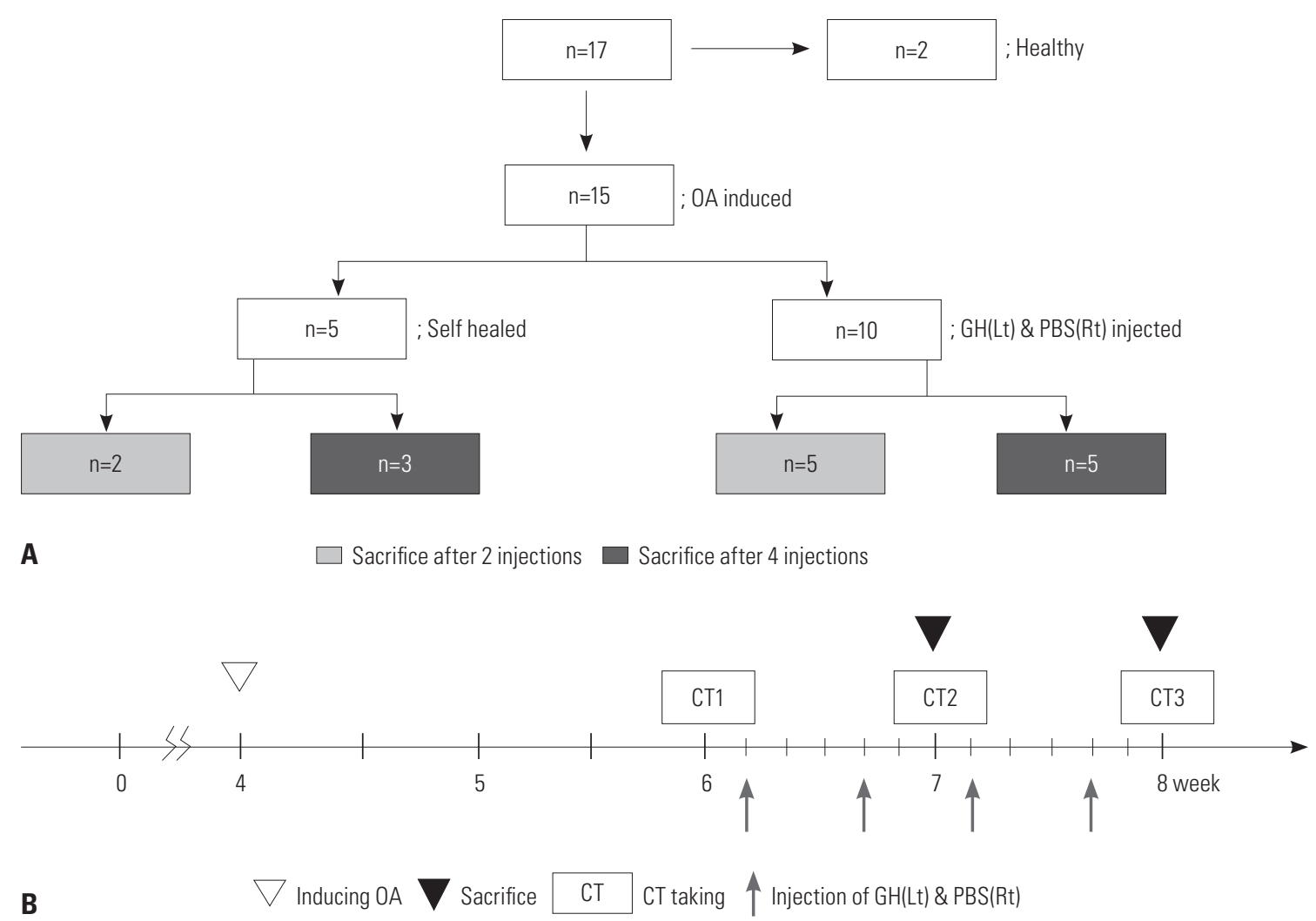

Fig. 1. Diagram of the study design. $\mathrm{OA}$, osteoarthritis; $\mathrm{GH}$, growth hormone; $\mathrm{PBS}$, phosphate buffered saline; $\mathrm{CT}$, micro-computed tomography.

chrome staining.

\section{Cartilaginous OA score}

OA cartilage histopathology was assessed using Masson's trichrome stained samples. Histological OA grading of cartilage degradation was performed as described previously ${ }^{21}: 0$, normal; 1, surface irregularities; 2 , pannus and surface irregularities; 3 , clefts to transitional zones; 4 , clefts to radial zones; 5 , clefts to calcified zones; and 6, complete disorganization. A pathologist who was blinded to the experiment graded all cartilage sections.

\section{Blood screening for growth hormone and insulin-like growth factor-1}

Enzyme-linked immunohistochemistry (ELISA) tests (R\&D Systems, Inc., Minneapolis, MN, USA) were used to measure GH and IGF-1 levels in serum and synovial fluid. Whole-blood samples were collected into tubes and centrifuged at $13000 \mathrm{rpm}$ for $10 \mathrm{~min}$ at $4^{\circ} \mathrm{C}$. Aliquots of serum and synovial fluid were stored at $-80^{\circ} \mathrm{C}$ until ready for analysis. The assay was conducted according to the manufacturer's recommended protocols. Standard serum diluents and synovial fluid were then added in duplicate to individual wells and incubated for $2 \mathrm{~h}$ at $37^{\circ} \mathrm{C}$. After washing the plates five times with wash buffer, 100 $\mu \mathrm{L}$ of conjugate was added to each well and incubated for $2 \mathrm{~h}$ at room temperature. After incubation, the plates were washed another five times with wash buffer. Substrate solution was then added to the wells and incubated for $30 \mathrm{~min}$ at room temperature in the dark. The reaction was stopped with $100 \mu \mathrm{L}$ of $\mathrm{H}_{2} \mathrm{SO}_{4}$ solution (Merck, Darmstadt, Germany). The absorbance was determined using a spectrophotometric plate reader (VersaMax Microplate Reader; Molecular Reader, Toronto, Canada) at a $40-n m$ wavelength.

\section{Statistical analysis}

All statistical analyses were performed using IBM SPSS version 22.0 (IBM Corp., Armonk, NY, USA). The Mann-Whitney test was used to determine significant differences in mean values of two independent groups. The Kruskal-Wallis test was used to compare mean values or scores for changes over three independent groups. The Wilcoxon signed-rank test was used to confirm statistically significant changes in the OA scores of respective samples before and after treatment. Differences with $p<0.05$ were considered statistically significant.

\section{RESULTS}

\section{MIA successfully induces $\mathrm{OA}$ in the TMJ of rats}

As shown in Fig. 2, the normal controls exhibited sound cortical lining, as well as normal trabecular patterns on micro-CT images (Fig. 2A). The intactness of the structures, including fibrous covering, fibrocartilage, and osteochondral tissue, were histologically confirmed in the tissue sections (Masson's tri- 

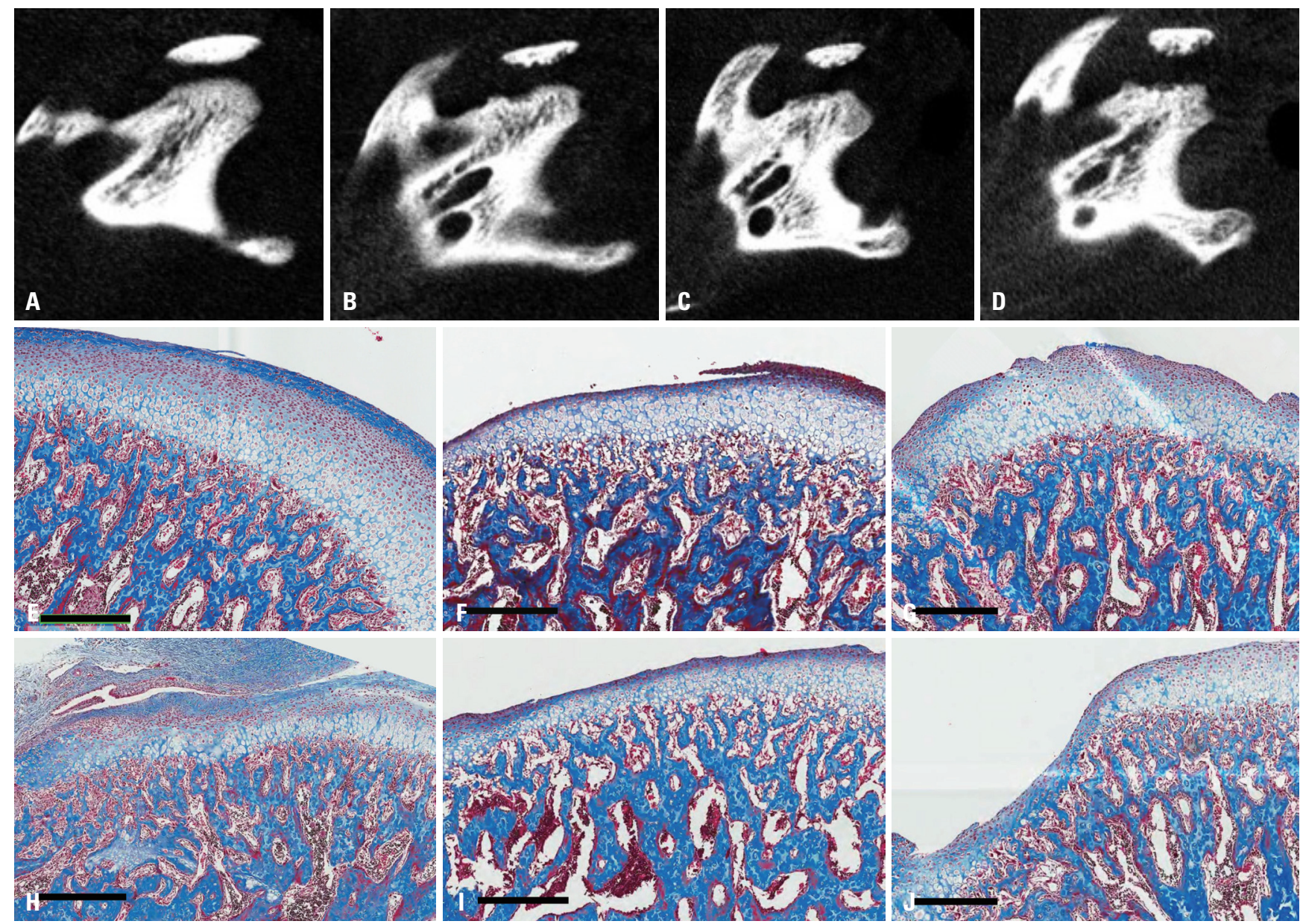

Fig. 2. OA scoring of the bone (micro-CT) and cartilage (histologic finding). (A-D) Central sagittal micro-CT images of erosive condyles to score subchondral bone: bony OA score=0 (A, None), 1 (B, Minor), 2 (C, Moderate), and 3 (D, Severe). (E-J) Histologic findings (Masson's trichrome staining): healthy $(\mathrm{E})$, cartilaginous $0 A$ score $=1(\mathrm{~F}), 2(\mathrm{G}), 3(\mathrm{H}), 4(\mathrm{I})$, and $5(\mathrm{~J})$. The black scale bar in the histological images represents $300 \mu \mathrm{m}$. $0 \mathrm{~A}, 0$ steoarthritis; CT, computed tomography.

chrome stain). Normal tissue consisted of five layers: fibrous covering, proliferative region, fibrocartilage region, calcified cartilage region, and subchondral bony region (Fig. 2E). When micro-CT images were taken at 2 weeks after a $50-\mu \mathrm{L}$ intra-articular injection of $1 \mathrm{mg}$ of MIA (CT1), erosive OA stages could be observed (Fig. 2B-D). Fig. 2 depicts the degrees of increasing TMJ-OA and relative OA scores: the micro-CT images show bony OA scores, while the histologic views represent cartilaginous $\mathrm{OA}$ scores.

Fig. 2J denotes a representative histological view showing a cartilaginous OA score of 5. Extensive loss of fibrous covering with only small parts remaining were seen. The underlying cartilage was lost, and subchondral bone was exposed. Highpower microscopy revealed bone formation in the areas below the destructed joint, although it was not calcified; rather, it was in an immature state, with the central part of the joint showing extensive trabecular destruction replaced with fibrous tissue. Furthermore, the remaining fibrocartilage and underlying cartilage were thin and irregular in shape, compared to the control group. In the areas of bone exposure, the fibrous covering, fibrocartilage, and underlying cartilage were all destroyed and showed increased numbers of fibroblasts, osteoclasts, and inflammatory cells, with less trabecular calcification (Fig. 2J; cartilaginous OA score=5).

\section{Local GH injections increase IGF-1 levels in synovial fluid, but not throughout the length of the condyle} Changes in GH and IGF-1 levels were observed on ELISA (Figs. 3 and 4). Significant changes in GH occurred in the blood ( $p=$ 0.003) (Fig. 3A), where IGF-1 levels remained unaffected (Fig. $3 \mathrm{~B})$. There were no changes in condylar length among the healthy, OA-induced only, and GH-injected groups (data not shown). When we injected GH directly into the TMJs, we found that IGF-1 levels responded positively in the synovial fluid ( $p=0.016)$ (Fig. 4B); however, there were no statistically significant changes in GH levels (Fig. 4A).

\section{Locally injected GH improves OA scores for} subchondral bone and cartilage of the TMJ

To determine the effect of GH in bony remodeling of arthritic 

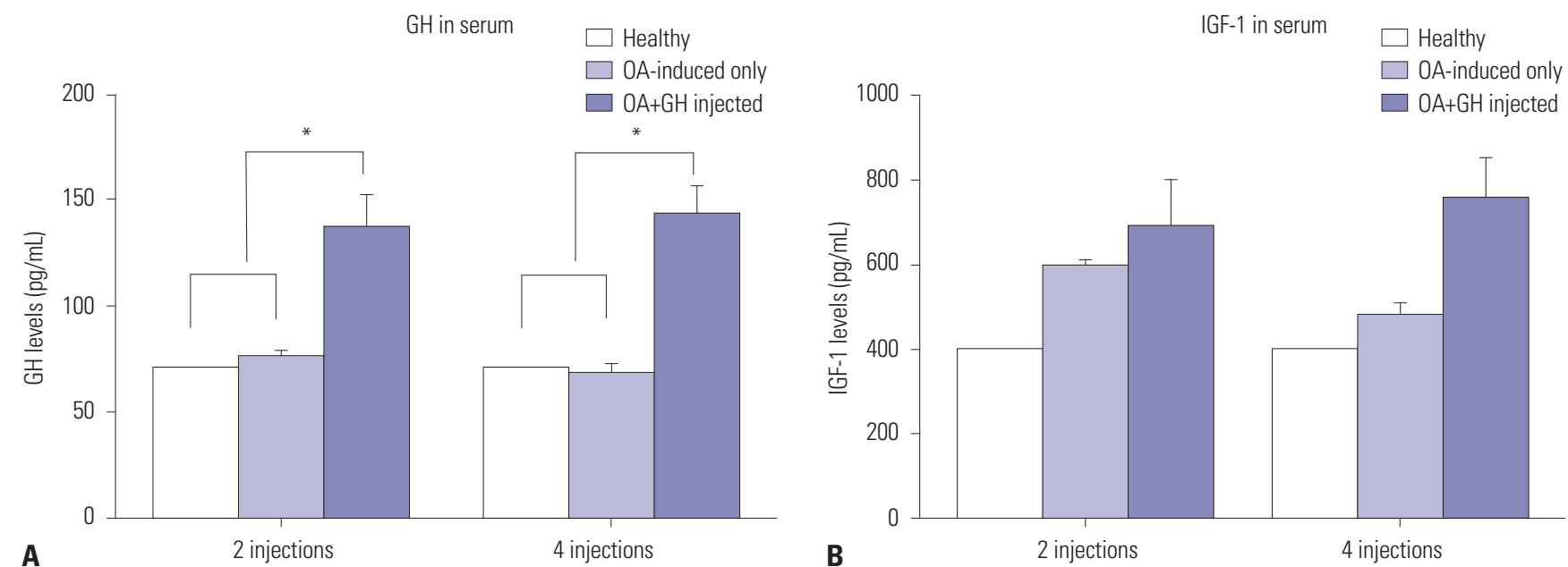

Fig. 3. GH injections into the TMJ increase serum GH levels, but not IGF-1 levels. After two and four injections of PBS(Rt) or GH(Lt) into the TMJ, GH (A) and IGF-1 (B) concentrations in serum were measured using ELISA. Healthy $(n=2), 0 A$-induced only ( $n=5$, allowed to self-heal after induction of $\mathrm{OA}$ ), and $\mathrm{OA}+\mathrm{GH}$ injected $(\mathrm{n}=10)$. ${ }^{*} p=0.003$ (Mann-Whitney test). $\mathrm{GH}$, growth hormone; IGF-1, insulin-like growth factor 1; PBS, phosphate buffered saline; TMJ, temporomandibular joint; ELISA, enzyme-linked immunosorbent assay; OA, osteoarthritis.
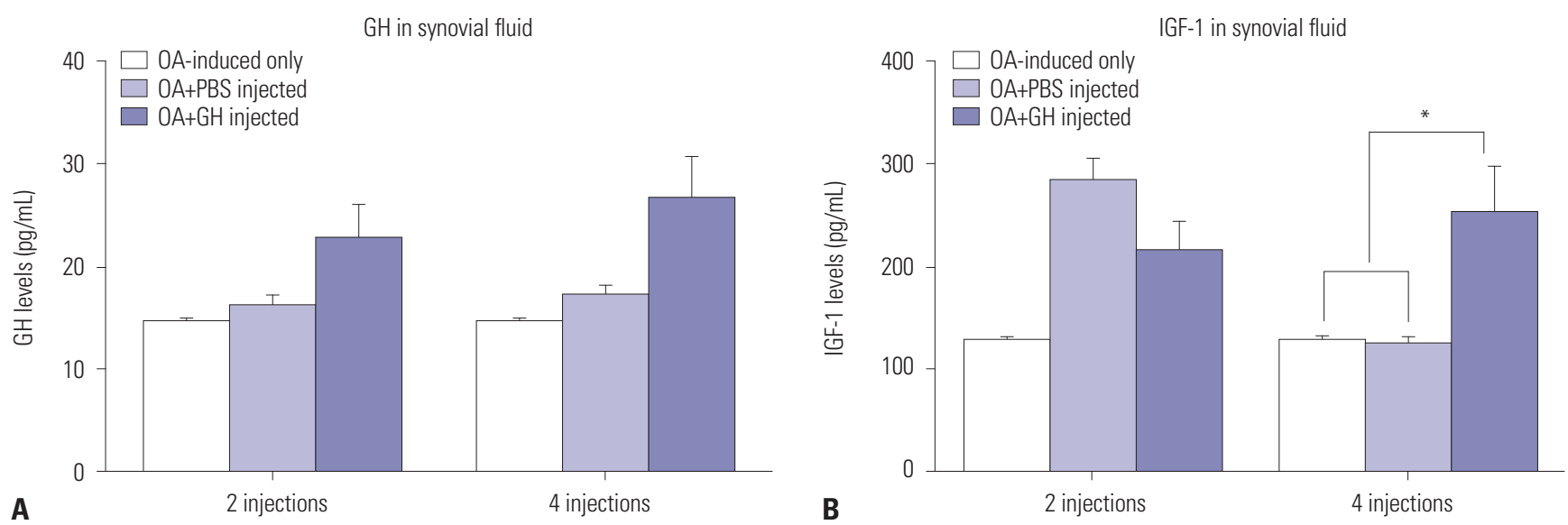

Fig. 4. GH injections into the TMJ increase IGF-1 levels in synovial fluid. After two and four injections of PBS(Rt) or GH(Lt) into the TMJ, the GH (A) and IGF-1 (B) concentrations in synovial fluid from the TMJ were measured by ELISA. OA-induced only ( $\mathrm{n}=5$, allowed to self-heal after induction of $0 A$ ), $0 A+P B S$ injected $(n=10)$, and $0 A+G H$ injected $(n=10) .{ }^{*} p=0.016$ (Kruskal-Wallis test). GH, growth hormone; TMJ, temporomandibular joint; IGF-1, insulin-like growth factor 1; PBS, phosphate buffered saline; ELISA, enzyme-linked immunosorbent assay; OA, osteoarthritis.

TMJ, OA bone scores were analyzed using micro-CT (Fig. 2). Once MIA-induced OA was confirmed (CT1), images were taken after two GH injections (CT2) and after four GH injections (CT3).

Although scores varied in the degree of induced OA between subjects, the average MIA-induced OA score was 1.55 before starting GH injection. In rats with PBS-injected TMJs, only a small change in average OA scores was observed. On the individual level, most of the subjects showed a slight reduction in OA scores. However, two subjects actually showed an increase in OA scores. On the other hand, GH-injected TMJs showed marked reductions in average OA scores at both after two (Fig. 5A) and four (Fig. 5B) injections, and OA scores in the subchondral bone were significantly better for the TMJs from the $\mathrm{GH}$-injected group, compared to those from the PBS-injected group (Wilcoxon signed-rank test, $p=0.010$ ).
Histological OA grading of cartilage degradation via Masson's trichrome staining was performed as described previously. ${ }^{21}$ As changes in cartilage were difficult to verify on the CT images, confirmation could only be made after the subjects were euthanized at the end of the experiment (Fig. 2E-J). There was only a small difference in cartilaginous OA scores from PBS-injected and GH-injected TMJs after two injections. However, there was a significant difference in OA scores for the PBSinjected and GH-injected TMJ groups after four injections ( $p<$ 0.01) (Fig. 6B).

Fig. 6 shows CT and Masson's trichrome stained tissue images of test subject number 3 , which was treated with four injections of GH in the left TMJ (Fig 6A-a, b, c) and PBS in the right TMJ (Fig 6A-d, e, f). Generalized condylar head OA (score 3 ) is visible in Fig. 6A-a; this score was much higher than that shown in Fig. 6A-d (score 1), indicating that the defect was 

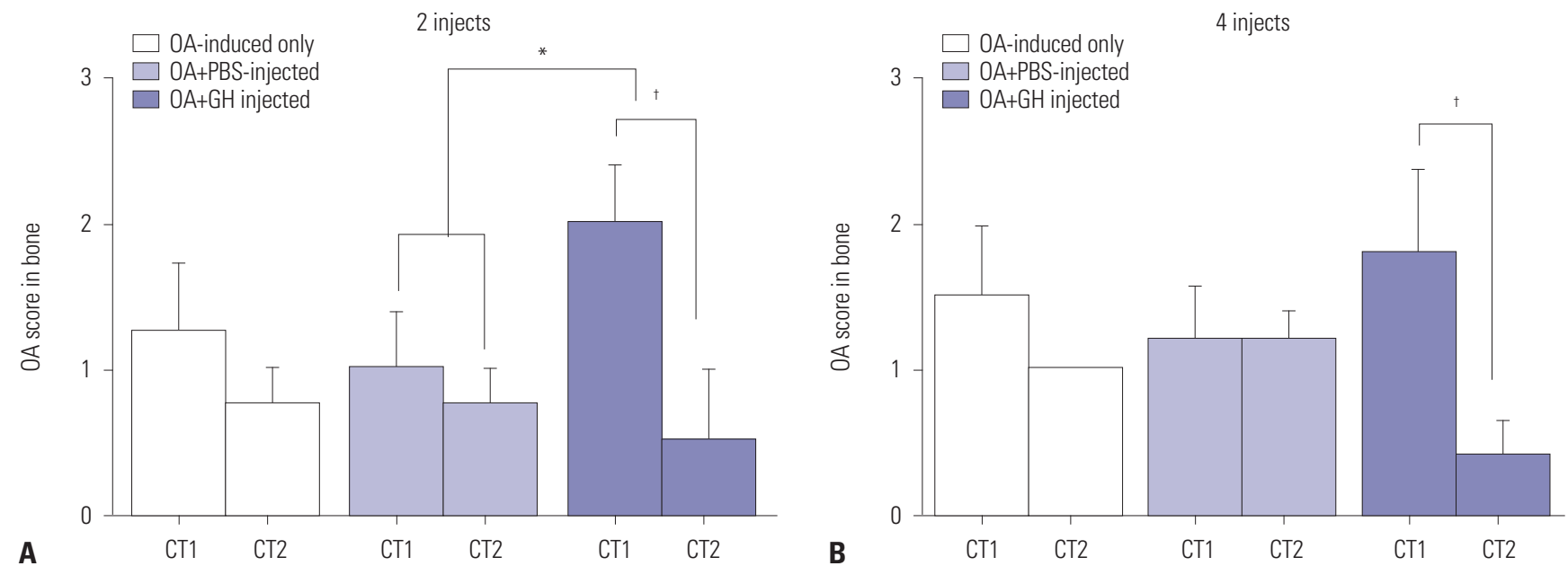

Fig. 5. Local injection of $\mathrm{GH}$ ameliorates $\mathrm{OA}$ joint in subchondral bone of the temporomandibular. OA scores were determined using in vivo micro-CT images performed before PBS or GH administration and after two $(A)$ and four $(B)$ injections of PBS or GH. OA-induced only ( $n=5$, allowed to self-heal after induction of $\mathrm{OA}), 0 \mathrm{~A}+\mathrm{PBS}$ injected $(\mathrm{n}=10)$, and $\mathrm{OA}+\mathrm{GH}$ injected $(\mathrm{n}=10)$. ${ }^{*}$ Differences were statistically significant at $p<0.05$, ${ }^{\dagger} D$ ifferences were statistically significant at $p<0.01$. OA, osteoarthritis; CT, computed tomography; CT1, OA score observed on initial CT images immediately after OA was induced; CT2, OA score observed on CT images after two injections; CT3, OA score observed on CT images after four injections; GH, growth hormone; TMJ-OA, osteoarthritis of the temporomandibular joint; micro-CT, micro-computed tomography; PBS, phosphate buffered saline.

much larger at the initial stage. However, images taken after four GH injections revealed that the score had decreased to 1 (Fig. 6A-b). As seen in Fig. 6A-c, the repaired condyle after the GH injection exhibited the maintenance of all cellular layers and ossification of about $10 \%$ of the cartilage tissue. On the other hand, the OA score of the TMJ treated with PBS was recorded as 1 before the experiment (Fig. 6A-d), and after four PBS injections, the score rose to 2, showing that the OA had worsened (Fig. 6A-e). The histological findings showed that approximately $50 \%$ of the TMJ was composed of bone and reparative tissue, and the remaining $50 \%$ showed all cellular layers, although thinning of the hypertrophic layer was observed (Fig. 6A-f).

\section{DISCUSSION}

Although several hypotheses for OA progression have been proposed, in general, changes are thought to initiate from cartilage, followed by changes in subchondral bone through cartilage and bone interaction. ${ }^{14}$ The primary aim of TMJ-OA treatment is reducing TMJ overloading and pain management by eliminating inflammatory responses in the capsular space. OA in middle-aged to older adults appears to be accompanied by degenerative joint changes, whereas $\mathrm{OA}$ in adolescents does not display a degenerative etiology. ${ }^{4}$ Therefore, many attempts have been made to determine the etiology of $\mathrm{OA}$ in accordance with etiologic factors, such as genetic factors, parafunctional habits, occlusal disturbance, and sudden dental treatment, ${ }^{1,22}$ and to develop treatments to eliminate these etiologic factors. Treatment options include physical therapy, medication, stabilization splint therapy, and behavioral therapy. These treat- ments are dependent on recovery of the condyle through the physiologic remodeling process, and their results depend on individual's cell activity and OA severity. ${ }^{23}$ There have been numerous attempts to assist with the regeneration of the cells that form the condyle. Among them, the most common for direct intra-articular injection include hyaluronic acid, ${ }^{24}$ steroids, ${ }^{25}$ and IGF- $1{ }^{26}$

GH is known to play a definite role in bone growth, and its efficacy has been proven on many occasions. Moreover, it was long ago suggested that GH directly stimulates pre-chondrocyte generation in the germinal cell layer of the growth plate, leading to proliferation of chondrocytes. GH can also activate IGF-1 indirectly to further stimulate chondrocyte proliferation. ${ }^{27}$ One study involving hypophysectomized rats showed that systemic administration or local injection of GH could stimulate chondrocyte growth in the epiphyseal growth plate. ${ }^{28}$ Another found that GH improved chondrocyte generation and proliferation independent of IGF-1 when it was injected into experimental animals that could not produce IGF- $1{ }^{29}$

Normal condylar tissue consists of five layers: fibrous covering, proliferative region, fibrocartilage region, calcified cartilage region, and subchondral bony region (Fig. 2E). The MIA used in this study is a representative substance that inhibits chondrocyte metabolism, thereby inducing apoptosis and reducing cartilage matrix. When injected intra-articularly, irregular surfaces, loss of superficial cell layers, and generalized growth inhibition can be induced. ${ }^{30}$ The histological findings of this experiment, such as the loss of superficial cell layers, irregularity in the fibrocartilage region, destruction of trabeculae, and fibrous tissue proliferation into the destructed trabeculae space, were consistent with the results of previous studies. Extensive loss of the fibrous covering with only small parts re- 

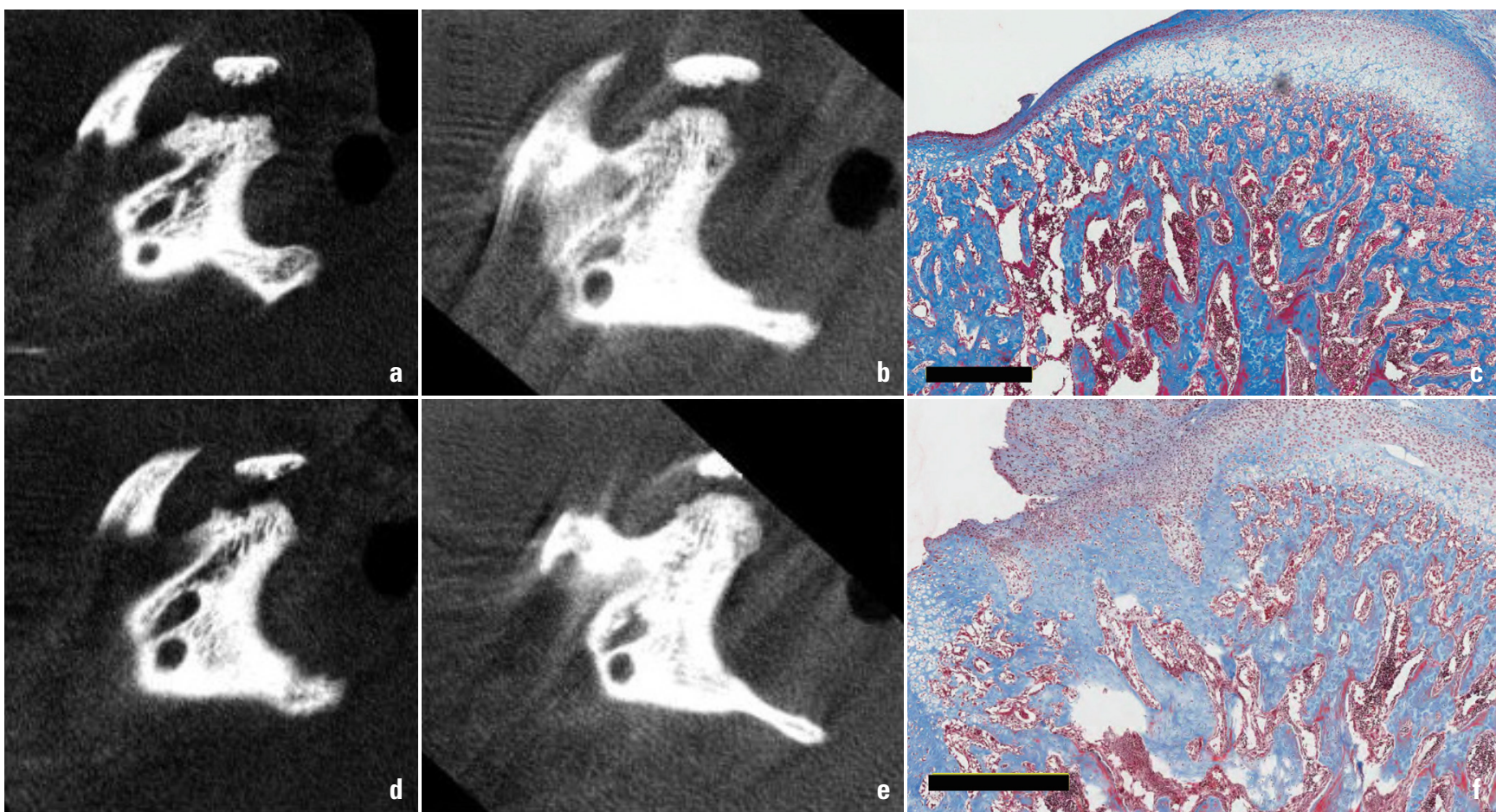

A

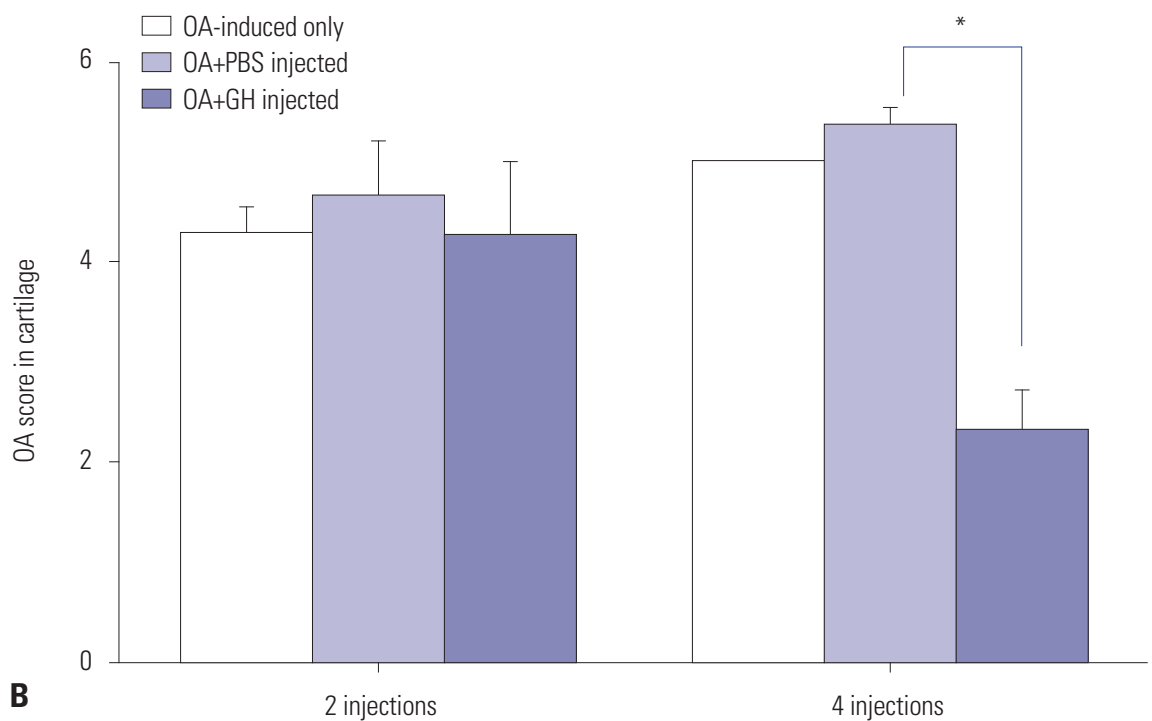

Fig. 6. GH injection into the MIA-induced $\mathrm{OA}$ of the TMJ area significantly improves $\mathrm{OA}$ scores in the cartilage. (A) All images were obtained from one rat after four injections of GH in the left TMJ $(a, b, c)$ and PBS in the right TMJ (d, e, f). TMJ-OA was induced by MIA injection, and micro-CT images (CT1) were acquired at 2 weeks after MIA injection to confirm OA induction (a, d). Treatment with GH (b) or PBS (e) injection was administered four times, and images (CT3) were taken after administration. Histologic findings of panels (b) and (e) were observed using Masson's trichrome stain respective to (c) and (f). The black scale bar in the histological images represents $500 \mu \mathrm{m}$. (B) Histologic OA scores were compared between PBS- and GH-injected groups. OA-induced only ( $n=5$, allowed to self-heal after induction of $O A), O A+P B S$ injected $(n=10)$, and $0 A+G H$ injected $(n=10)$. ${ }^{*} p=0.004$ (Mann-Whitney test). OA, osteoarthritis; GH, growth hormone; MIA, monosodium iodoacetate; TMJ-OA, osteoarthritis of the temporomandibular joint; micro-CT, micro-computed tomography; PBS, phosphate buffered saline.

maining was seen in induced TMJ-OA. The underlying cartilage was lost, and subchondral bone was exposed (Fig. 2I and J). Furthermore, MIA-induced TMJ-OA showed irregular surfaces, erosions which are consistent findings with those of the CT images of highly advanced OA patients. ${ }^{18}$ Thus, it was deemed a good method to model human TMJ-OA (Fig. 2D).
According to Wang, et al., ${ }^{18}$ starting 3 days after MIA injection, chondrocytes show nuclear condensation and enter apoptosis. Also, TUNEL-positive chondrocytes nearly disappear by 1 week after the injection; after 2 weeks, the shape of subchondral bone is somewhat maintained and erosion becomes visible on CT images. In this study, GH was injected 2 weeks after MIA 
administration, because this was the point at which the focal erosion is seen most frequently in adolescents.

In the OA+PBS group, IGF-1 levels in synovial fluid were higher after two injections than after four. We assumed that IGF-1 was secreted temporarily into the damaged areas for GH-independent healing in the body. This may explain why there was transient expression of IGF- 1 in joints that received only PBS after the induction of $\mathrm{OA}$ (Fig. 4B). This same result was observed in a previous study, which demonstrated that IGF-1 in the synovial fluid can be generated in response to damage. ${ }^{31}$ When OA is progressing, a temporary increase in IGF-1 levels in synovial fluid is assumed due to release from chondrocytes independent from serum GH.

In this study, after GH injection, the amount of GH in the blood increased (Fig. 3A). This increase in serum GH could be due to leakage of injected GH from the articular space. The increase in IGF-1 levels in the blood was not significant after the GH injection (Fig. 3B). However, a distinctive increase was observed in the synovial fluid, and this was higher in the second week after four injections (Fig. 4B). IGF-1 released from the damaged joint surface could induce tissue healing, although in situations in which continuous secretion did not occur, the GH injections maintained IGF-1 concentrations. This supports the notion that using GH could be an effective treatment option for OA.

In this study, local GH injection improved subchondral bone OA scores in damaged TMJs (Fig. 5), and a marked improvement in cartilaginous OA scores was observed after four GH injections (Fig. 6B). From this, a hypothesis can be made to suggest that GH either directly acts on chondrocytes for regeneration or improves the inflammatory response, therefore providing the right environment for cartilage regeneration. GH is known to stimulate cells directly and indirectly by controlling IGF-1 locally or systemically to achieve a major role in linear bone growth. ${ }^{7}$ Visnapuu, et al. ${ }^{32}$ reported that GH receptors were located in the mineralized area of the rat TMJ and that IGF-1 receptors were detected in the cartilage layer of the condyle. In this study, locally injected GH was able to bind to receptors in the exposed subchondral bone. After binding to its chondrocyte membrane receptor, GH activates a number of well-recognized intracellular signal transduction pathways that regenerate the transcription of GH target genes, including IGF-1, to orchestrate the array of chondrocyte events necessary for linear bone growth to proceed. ${ }^{33}$ Chondrocytes within the growth plate proceed through proliferation, differentiation, and maturation stages while maintaining their spatially fixed locations. ${ }^{34}$ This acts as an important key factor to form various conditions permissive to vascular infiltration for new bone growth in the chondro-osseous junction. ${ }^{34}$ Although GH levels in the blood increased in the GH injected group, no difference in condylar length was observed among the normal, OA-induced only, and GH injected groups (data not shown). These results showed that while systemic GH concentration in-

creases, this increase does not affect overall mandibular growth.

Studies have reported that OA patients show significantly elevated levels of GH in the basal serum, compared to healthy individuals, ${ }^{35}$ while other have shown that serum GH levels decrease to normal when pain and swelling are reduced by TMJ treatment, including that with nonsteroidal anti-inflammatory drugs. ${ }^{36}$ Thus, while the interaction between GH and inflammation is clearly evident, it has not yet been clarified. Moreover, the role of GH or IGF-1 cannot be clearly distinguished in these processes. It is true that GH stimulates IGF-1 production $^{37}$; however, one study showed that GH does not function in mutant mice lacking IGF- $1{ }^{38}$ It is possible that GH and IGF-1 have independent and shared functions, as growth retardation of double $\mathrm{ghr} / \mathrm{igf-lr}$ mutants is more severe than that observed with the ghr mutant or igf- 1 null mutant. ${ }^{39}$

In this study, the small numbers of animals would be unable to represent all aspects of $\mathrm{OA}$, and the severity of induced OA differed among the animals. Therefore, the same level of improvement could not be observed. However, when GH is injected locally, it can play a role in improving $\mathrm{OA}$ scores in rat TMJs for both cartilage and subchondral bone without affecting systemic bone growth as seen in this study. The local injection of GH positively affects subchondral bone and can repair the damaged cartilaginous layer. Therefore, we deemed local injections of GH to be useful in treating TMJ-OA in early stages due to its ability to maintain a cartilaginous layer and thus prevent a change in subchondral bone shape. Notwithstanding, further studies are needed to explore the different responses among animals and results of long-term administration of $\mathrm{GH}$.

\section{ACKNOWLEDGEMENTS}

This study was supported by the Dental Research Institute (PNUDH DRI-2014-01), Pusan National University Dental Hospital.

\section{AUTHOR CONTRIBUTIONS}

Conceptualization: Sung-Hee Jeong. Data curation: Jin-Hwa Kim, JiSu Kim, and Eun-gyo Jeong. Formal analysis: Sun-Nyoung Yu. Funding acquisition: Sung-Hee Jeong. Investigation: Sun-Nyoung Yu. Methodology: Yang Mi Park. Project administration: Hye-Mi Jeon, Yong-Woo Ahn, and Kyung-Hee Kim. Resources: Jun-Young Heo. Software: Jin-Hwa Kim and Eun-gyo Jeong. Supervision: Sung-Hee Jeong. Validation: Hae Ryoun Park . Visualization: Sun-Nyoung Yu. Writing_original draft: Sung-Hee Jeong and Soo-Min Ok. Writingreview \& editing: Sung-Hee Jeong, Soo-Min Ok, Soon-Cheol Ahn, and Hae Ryoun Park. Approval of final manuscript: all authors.

\section{ORCID iDs}

Soo-Min Ok Jin-Hwa Kim Ji-Su Kim Eun-gyo Jeong
https://orcid.org/0000-0003-1776-371X https://orcid.org/0000-0002-7263-6185 https://orcid.org/0000-0003-4744-8323 https://orcid.org/0000-0002-4468-9175 
Yang Mi Park Hye-Mi Jeon Jun-Young Heo Yong-Woo Ahn Sun-Nyoung Yu Hae Ryoun Park Kyung-Hee Kim Soon-Cheol Ahn Sung-Hee Jeong https://orcid.org/0000-0002-4508-2543 https://orcid.org/0000-0003-0007-5662 https://orcid.org/0000-0002-4617-1759 https://orcid.org/0000-0002-2197-0394 https://orcid.org/0000-0002-2424-7003 https://orcid.org/0000-0003-1908-0824 https://orcid.org/0000-0003-2922-6452 https://orcid.org/0000-0002-5826-6392 https://orcid.org/0000-0002-6296-4775

\section{REFERENCES}

1. Su N, Liu Y, Yang X, Shen J, Wang H. Correlation between oral health-related quality of life and clinical dysfunction index in patients with temporomandibular joint osteoarthritis. J Oral Sci 2016;58:483-90.

2. Billiau AD, Hu Y, Verdonck A, Carels C, Wouters C. Temporomandibular joint arthritis in juvenile idiopathic arthritis: prevalence, clinical and radiological signs, and relation to dentofacial morphology. J Rheumatol 2007;34:1925-33.

3. Bilgiç F, Gelgör İE. Prevalence of temporomandibular dysfunction and its association with malocclusion in children: an epidemiologic study. J Clin Pediatr Dent 2017;41:161-5.

4. Ok SM, Kim CY, Jeong SH, Ahn YW, Ko MY. Comparative analysis: the patterns of temporomandibular disorder among adolescents. J Oral Med Pain 2012;37:47-59.

5. Kajii TS, Fujita T, Sakaguchi Y, Shimada K. Osseous changes of the mandibular condyle affect backward-rotation of the mandibular ramus in Angle Class II orthodontic patients with idiopathic condylar resorption of the temporomandibular joint. Cranio 2019;37: 264-71.

6. Wolford LM, Reiche-Fischel O, Mehra P. Changes in temporomandibular joint dysfunction after orthognathic surgery. J Oral Maxillofac Surg 2003;61:655-60.

7. Nilsson O, Marino R, De Luca F, Phillip M, Baron J. Endocrine regulation of the growth plate. Horm Res 2005;64:157-65.

8. Nemirovskiy O, Zheng YJ, Tung D, Korniski B, Settle S, Skepner A, et al. Pharmacokinetic/pharmacodynamic (PK/PD) differentiation of native and PEGylated recombinant human growth hormone (rhGH and PEG-rhGH) in the rat model of osteoarthritis. Xenobiotica 2010;40:586-92.

9. Bail H, Klein P, Kolbeck S, Krummrey G, Weiler A, Schmidmaier $\mathrm{G}$, et al. Systemic application of growth hormone enhances the early healing phase of osteochondral defects--a preliminary study in micropigs. Bone 2003;32:457-67.

10. Reinecke M, Schmid AC, Heyberger-Meyer B, Hunziker EB, Zapf J. Effect of growth hormone and insulin-like growth factor I (IGF-I) on the expression of IGF-I messenger ribonucleic acid and peptide in rat tibial growth plate and articular chondrocytes in vivo. Endocrinology 2000;141:2847-53.

11. Dunn AR. Morphoangiogenesis: a unique action of growth hormone. Microvasc Res 2002;63:295-303.

12. Kim SB, Kwon DR, Kwak H, Shin YB, Han HJ, Lee JH, et al. Additive effects of intra-articular injection of growth hormone and hyaluronic acid in rabbit model of collagenase-induced osteoarthritis. J Korean Med Sci 2010;25:776-80.

13. Laron Z. Insulin-like growth factor 1 (IGF-1): a growth hormone. Mol Pathol 2001;54:311-6.

14. Wei FY, Lee JK, Wei L, Qu F, Zhang JZ. Correlation of insulin-like growth factor 1 and osteoarthritic cartilage degradation: a spontaneous osteoarthritis in guinea-pig. Eur Rev Med Pharmacol Sci 2017;21:4493-500.

15. Daughaday WH. Growth hormone axis overview--somatomedin hypothesis. Pediatr Nephrol 2000;14:537-40.

16. Demarquay D, Dumontier MF, Bourguignon J, Hintz RL, Corvol MT. Stimulation by GH of IGF1 proforms synthesized by rabbit chondrocytes cultured with bFGF in serum-free medium. Exp Cell Res 1992;202:412-22.

17. Páramo C, Fluiters E, de la Fuente J, Andrade A, García-Mayor RV. Monitoring of treatment success in patients with acromegaly: the value of serum insulin-like growth factor binding protein-3 and serum leptin measurements in comparison to plasma insulinlike growth factor I determination. Metabolism 2001;50:1117-21.

18. Wang XD, Kou XX, He DQ, Zeng MM, Meng Z, Bi RY, et al. Progression of cartilage degradation, bone resorption and pain in rat temporomandibular joint osteoarthritis induced by injection of iodoacetate. PLoS One 2012;7:e45036.

19. Khan I, El-Kadi AO, El-Bialy T. Effects of growth hormone and ultrasound on mandibular growth in rats: MicroCT and toxicity analyses. Arch Oral Biol 2013;58:1217-24.

20. Møystad A, Mork-Knutsen BB, Bjørnland T. Injection of sodium hyaluronate compared to a corticosteroid in the treatment of patients with temporomandibular joint osteoarthritis: a CT evaluation. Oral Surg Oral Med Oral Pathol Oral Radiol Endod 2008;105: e53-60.

21. Kwon DR, Park GY, Lee SU. The effects of intra-articular plateletrich plasma injection according to the severity of collagenase-induced knee osteoarthritis in a rabbit model. Ann Rehabil Med 2012;36:458-65.

22. Su N, Liu Y, Yang X, Shen J, Wang H. Association of malocclusion, self-reported bruxism and chewing-side preference with oral health-related quality of life in patients with temporomandibular joint osteoarthritis. Int Dent J 2018;68:97-104.

23. Wahlund K, Larsson B. Long-term treatment outcome for adolescents with temporomandibular pain. Acta Odontol Scand 2018;76: 153-60.

24. Iturriaga V, Bornhardt T, Manterola C, Brebi P. Effect of hyaluronic acid on the regulation of inflammatory mediators in osteoarthritis of the temporomandibular joint: a systematic review. Int J Oral Maxillofac Surg 2017;46:590-5.

25. Machado E, Bonotto D, Cunali PA. Intra-articular injections with corticosteroids and sodium hyaluronate for treating temporomandibular joint disorders: a systematic review. Dental Press J Orthod 2013;18:128-33.

26. Liu XW, Hu J, Man C, Zhang B, Ma YQ, Zhu SS. Insulin-like growth factor-1 suspended in hyaluronan improves cartilage and subchondral cancellous bone repair in osteoarthritis of temporomandibular joint. Int J Oral Maxillofac Surg 2011;40:184-90.

27. Ohlsson C, Bengtsson BA, Isaksson OG, Andreassen TT, Slootweg MC. Growth hormone and bone. Endocr Rev 1998;19:55-79.

28. Lewinson D, Harel Z, Shenzer P, Silbermann M, Hochberg Z. Effect of thyroid hormone and growth hormone on recovery from hypothyroidism of epiphyseal growth plate cartilage and its adjacent bone. Endocrinology 1989;124:937-45.

29. Wang J, Zhou J, Cheng CM, Kopchick JJ, Bondy CA. Evidence supporting dual, IGF-I-independent and IGF-I-dependent, roles for GH in promoting longitudinal bone growth. J Endocrinol 2004; 180:247-55.

30. Güler N, Kürkçü M, Duygu G, Cam B. Sodium iodoacetate induced osteoarthrosis model in rabbit temporomandibular joint: CT and histological study (part I). Int J Oral Maxillofac Surg 2011;40: 1289-95.

31. Loeser RF, Pacione CA, Chubinskaya S. The combination of insulin-like growth factor 1 and osteogenic protein 1 promotes increased survival of and matrix synthesis by normal and osteoarthritic human articular chondrocytes. Arthritis Rheum 2003;48: 
2188-96.

32. Visnapuu V, Peltomäki T, Rönning O, Vahlberg T, Helenius H. Growth hormone and insulin-like growth factor I receptors in the temporomandibular joint of the rat. J Dent Res 2001;80:1903-7.

33. Waters MJ, Hoang HN, Fairlie DP, Pelekanos RA, Brown RJ. New insights into growth hormone action. J Mol Endocrinol 2006;36:1-7.

34. Farquharson C, Ahmed SF. Inflammation and linear bone growth: the inhibitory role of SOCS2 on GH/IGF-1 signaling. Pediatr Nephrol 2013;28:547-56.

35. Denko CW, Boja B, Moskowitz RW. Growth factors, insulin-like growth factor-1 and growth hormone, in synovial fluid and serum of patients with rheumatic disorders. Osteoarthritis Cartilage 1996; $4: 245-9$.
36. Denko CW, Malemud CJ. Role of the growth hormone/insulin-like growth factor-1 paracrine axis in rheumatic diseases. Semin Arthritis Rheum 2005;35:24-34.

37. Mathews LS, Hammer RE, Behringer RR, D'Ercole AJ, Bell GI, Brinster RL, et al. Growth enhancement of transgenic mice expressing human insulin-like growth factor I. Endocrinology 1988;123:282733.

38. Lupu F, Terwilliger JD, Lee K, Segre GV, Efstratiadis A. Roles of growth hormone and insulin-like growth factor 1 in mouse postnatal growth. Dev Biol 2001;229:141-62.

39. Ahmed SF, Farquharson C. The effect of GH and IGF1 on linear growth and skeletal development and their modulation by SOCS proteins. J Endocrinol 2010;206:249-59. 